\title{
Evaluation of Sympathetic Nerve Function Status in Hypertensive Patients
}

\author{
Tabassum $\mathrm{R}^{1}$, Mithun $\mathrm{S}^{2}$, Irfan $\mathrm{SMN}^{3}$
}

\begin{abstract}
Introduction: Hypertension is independent and the most powerful predictor of cardiovascular morbidity and mortality. Sympathetic overdrive may be related to essential hypertension. Heart Rate Variability (HRV) analysis is one of the most quantitative markers of autonomic activity in hypertensive patients.
\end{abstract}

Objective: To assess the cardiac autonomic nerve function status by heart rate variability in essential hypertension.

Materials and Methods: This cross sectional study was carried out to observe the HRV in 60 hypertensive male patients with age ranging from 40-60 years (group B) in the Department of Physiology, Bangabandhu Sheikh Mujib Medical University (BSMMU) from 1st July 2008 to 30th June 2009. Based on treatment, hypertensive patients were divided into $B_{1}$ (untreated patients on their 1st day of diagnosis) and $B_{2}$ (patients with antihypertensive therapy for less than 5 years). For comparison, 30 apparently healthy normotensive subjects (group A) matched by age sex were also studied. The study group was selected from the Out Patient Department of Cardiology, BSMMU, Dhaka and the control group was selected by personal contact. Heart Rate Variability parameters were assessed by Polygraph (RMS Polyrite D, version2.2) and for statistical analysis Independent sample t-test, One-way ANOVA test, Chi-square test and Pearson's correlation coefficient tests were done as applicable.

Results: LF/HF(Low Frequency/High Frequency) ratio and LF norm (normalized unit) were significantly $(p<0.001)$ higher and HF norm was significantly $(p<0.001)$ lower in untreated hypertensive patients in comparison to those of healthy normotensive subjects. parameters were observed between treated hypertensive and healthy control subjects. The LF/HF ratio and LF norm showed positive correlations and HF norm showed negative correlations with SBP (systolic blood pressure) and DBP (diastolic blood pressure) in both the hypertensive groups. However, all these findings were more marked in untreated hypertensive patients than those of treated group.

Conclusion: Hypertensive patients may have sympathovagal imbalance and is usually characterized by higher sympathetic as well as lower vagal modulation of the heart rate.

Key-words: Cardiovascular Morbidity and Mortality, Heart Rate Variability (HRV).

\section{Introduction}

Hypertension represents major risk factor for heart disease, stroke and kidney disease. The effective management of hypertension is therefore a primary health care objective in management of cardiovascular disease. Hypertension is an emerging health problem in Bangladesh among the cardiovascular diseases. The prevalence rate of hypertension is very high and more than $20 \%$ of the adults have hypertension ${ }^{1}$. Different studies on both animals and humans suggested that autonomic nervous system plays a crucial role in the development of hypertension ${ }^{2}$. The arterial baroreflex mechanism regulates blood pressure through reflex effects on the heart, resistance vessels and renal excretion of sodium and water ${ }^{3}$. There is evidence that faulty noradrenaline reuptake in the cardiac sympathetic nerves amplify the sympathetic neural signal in essential hypertensive patients ${ }^{4}$. Essential hypertension is treated with drugs which themselves modify the sympathoparasympathetic

1. Dr Rehnuma Tabassum, MBBS, MPhil, Associate Professor of Physiology, East West Medical College, Dhaka 2. Lt Col Sangita Mithun, MBBS, MPhil, Instructor of Physiology, AFMC, Dhaka 3. Lt Col S M Nurul Irfan, MBBS, MPH, Medical Officer, Base Bashar, Bangladesh Air Force, Dhaka. 
balance ${ }^{5,6}$. The analysis of heart rate variability is a powerful noninvasive tool for assessing cardiac autonomic nerve function status. It is an accurate, reliable, reproducible, yet simple to measure and process. The source of information for HRV is a continuous beat-by-beat measurement of interbeat intervals ${ }^{7}$. Cardiac autonomic nerve function both for sympathetic and parasympathetic activity were assessed by analysis of HRV parameters in time and frequency domain method.

For simple time domain measures, mean R-R interval and mean heart rate; for statistical time domain measures, SDNN (standard deviation of N-N interval) and RMSSD (square root of mean squared difference of successive NN intervals) and for frequency domain measures, total power, VLF power, LF power, HF power, LF norm, HF norm and LF/HF ratio were included. Several investigators from different countries observed that mean R-R interval was significantly lower along with significantly higher mean heart rate in untreated hypertensive than those of healthy control ${ }^{8-12}$. However, some of the investigators failed to find any significant differences of these parameters between these two groups ${ }^{10,13}$. Again many investigators documented lower values of SDNN and RMSSD in both untreated and treated hypertensive groups compared to those of normotensive subjects ${ }^{9-16}$.

Various researchers of different countries reported lower LF power and HF power in untreated patients than those of healthy control $^{7,10-12,14}$. Some other group of investigators also observed similar findings in treated group when compared to normotensive control $^{10,13,14,17}$. In our country hypertension is one of the common cardiovascular problems. Many studies have documented sympathetic hyperactivity as the principal underlying cause for hypertension. Therefore, qualitative measure of cardiac sympathetic nerve activity along with cardiovagal balance may throw some light on the role of autonomic modulation to develop hypertension and also change in the autonomic activity after treatment with antihypertensive therapy. So, the present study was carried out to observe the autonomic nerve function status in newly diagnosed untreated and treated hypertensive patients by analysis of HRV parameters.

\section{Materials and Methods}

This cross sectional study was carried out on 60 male hypertensive patients with age ranging from 40-60 years (group B) in the Department of Physiology, Bangabandhu Sheikh Mujib Medical University from July 2008 to June 2009. Based on treatment, hypertensive patients were divided into $B_{1}$ (untreated patients on their 1st day of diagnosis) and $B_{2}$ (patients with antihypertensive medication). For comparison, age, sex and BMI matched apparently healthy 30 normotensive subjects (group A) were also studied as control group. The study group was selected from the Out Patient Department of Cardiology, BSMMU, Dhaka and the control group was selected by personal contact. Both the groups were free from heart diseases, secondary hypertension, diabetes mellitus, renal diseases and psychic disorders.

After selection, the subjects were thoroughly informed about the objectives and detailed procedure of the study before examination and collection of blood sample. They were encouraged for voluntary participation and allowed freedom to withdraw from the study whenever they liked even after participation. When they agreed to enroll to the study, informed written consent were taken from them. For examination the subjects were advised to have their meal by 9:00 pm on the previous night, to remain free from any physical or mental stress, not to take sedatives or any drugs affecting central nervous system and to have a good sleep at night before the day of examination. The subjects were also asked to avoid tea or coffee at breakfast and to attend the Autonomic Nerve Function Test Laboratory in the Department of Physiology of Bangabandhu Sheikh Mujib Medical University between 9:00 a.m. to 11:00 a.m. on the day of examination. Then the subject was interviewed and detailed history regarding personal history, drug history, past medical history was taken to exclude the exclusion criteria. Then thorough physical examinations and anthropometric measurement including height and weight were taken. BMI was calculated from measured height and weight. All information were recorded in a prefixed questionnaire. Then he was kept under complete bed rest in supine position for 15-20 minutes in a cool and calm environment. During this period subject was advised 
not to talk, eat or drink and also not to perform physical or any mental activity, even sleep. Then all preparations for recording the Heart Rate Variability parameters were made by connecting the channels of ECG and a 5 minutes recording was taken in resting supine position and the HRV parameters were studied by both time and frequency domain

Heart Rate Variability parameters in time domain method like mean R-R interval, mean Heart Rate, SDNN and RMSSD were assessed by Polygraph (RMS Polyrite D, version 2.2) and for Statistical analyses One-way ANOVA test, independent sample t-test and Pearson's correlation coefficient test was done as applicable.

All the groups were matched for age and $\mathrm{BMI}$ (Table-I). The mean resting pulse rate $(p<0.05)$, systolic (SBP) and diastolic (DBP) blood pressures $(p<0.001)$ were significantly higher in group $B_{1}$ than those of group $A$ and $B_{2}$. But statistically no significant differences were observed when these parameters were compared in between group $A$ and group $B_{2}$ (Table-II).

Age and BMI in different groups $(n=90)$

\begin{tabular}{c|c|c|}
\hline & Age(years) & BMI $\left(\mathbf{k g} / \mathbf{m}^{2}\right)$ \\
\hline & $48.37 \pm 8.03$ & $24.46 \pm 3.28$ \\
\hline & $49.77 \pm 7.70$ & $25.37 \pm 2.93$ \\
\hline & $48.13 \pm 6.24$ & $23.47 \pm 4.16$ \\
\hline
\end{tabular}

Statistical analysis

\begin{tabular}{c|c|c|}
\hline & \multicolumn{2}{|c|}{ p values } \\
\hline $\mathrm{a}$ & $0.651 \mathrm{~ns}$ & $0.115 \mathrm{~ns}$ \\
\hline & $0.493 \mathrm{~ns}$ & $0.263 \mathrm{~ns}$ \\
\hline & $0.900 \mathrm{~ns}$ & $0.308 \mathrm{~ns}$ \\
\hline & $0.370 \mathrm{~ns}$ & $0.05 \mathrm{~ns}$ \\
\hline
\end{tabular}

Data were expressed as mean $\pm S D$. Statistical analysis were done by One-way ANOVA $^{a}$ and Independent sample t-test ${ }^{\mathrm{b}}$.

$\mathrm{BMI}=$ Body Mass Index,

ns $=p>0.05, n=$ number of subjects,

Group A: Apparently healthy normotensive (control),

Group B: Hypertensive (study group),

-Untreated, $B_{2}$-Treated.

Resting Pulse Rate and BP in different groups $(n=90)$

\begin{tabular}{c|c|c}
\hline$(\mathrm{bpm})$ & $\mathrm{SBP}(\mathrm{mm}$ of $\mathrm{Hg})$ & $\mathrm{DBP}(\mathrm{mm}$ of $\mathrm{Hg})$ \\
\hline $3 \pm 7.28$ & $112.67 \pm 10.73$ & $74.43 \pm 8.71$ \\
\hline \pm 10.22 & $156.33 \pm 16.18$ & $101.50 \pm 8.63$ \\
\hline \pm 9.09 & $117.83 \pm 14.24$ & $78.33 \pm 10.20$ \\
\hline
\end{tabular}

Statistical analysis

\begin{tabular}{|c|c|c|c}
\hline Groups & \multicolumn{3}{|c}{ p values } \\
\hline $\mathrm{A}_{\text {vs } \mathrm{B}_{1} \text { vs } \mathrm{B}_{2 \mathrm{a}}}$ & $0.036^{*}$ & $0.000^{\star * *}$ & $0.000^{\star * *}$ \\
\hline $\mathrm{A}$ vs $\mathrm{B}_{1 \mathrm{~b}}$ & $0.010^{*}$ & $0.000^{\star * *}$ & $0.000^{* * *}$ \\
\hline $\mathrm{A}$ vs $\mathrm{B}_{2 \mathrm{~b}}$ & $0.142 \mathrm{~ns}$ & $0.118 \mathrm{~ns}$ & $0.117 \mathrm{~ns}$ \\
\hline $\mathrm{B}_{1}$ vs $\mathrm{B}_{2 \mathrm{~b}}$ & $0.250 \mathrm{~ns}$ & $0.000^{\star * *}$ & $0.000^{* * *}$ \\
\hline
\end{tabular}

Data were expressed as mean $\pm S D$. Statistical analyses were done by One-way ANOVA ${ }^{a}$ and Independent sample t-test ${ }^{\mathrm{b}}$.

$\mathrm{SBP}=$ Systolic blood pressure,

$\mathrm{DBP}=$ Diastolic blood pressure.

Group A: Apparently healthy normotensive (control).

Group B: Hypertensive (study group)

$B_{1}$-Untreated, $B_{2}$-Treated.

${ }^{* * *}=p<0.001,{ }^{* *}=p<0.01,{ }^{*}=p<0.05, n s=p>0.05$,

$n=$ number of subjects.

The mean R-R interval was significantly lower $(p<0.01)$ and mean heart rate was significantly higher $(p<0.01)$ in group $B_{1}$ than those of group $A$. But statistically no significant differences were observed when these parameters were compared in between group $B_{1}$ and $B_{2}$ and between group $A$ and $\mathrm{B}_{2}$ (Table-III).

Mean SDNN and RMSSD were significantly $(p<0.001)$ lower in group $B_{1}$ compared to those of group $A$ and group $B_{2}$ except SDNN which was though lower than group $B_{2}$ but statistically the differences were not significant. Again, the difference of these values were significantly $(p<0.001)$ lower in group $B_{2}$ than those of group $A$ (Table-III).

Table-III: Time domain measures of HRV in different groups $(n=90)$

\begin{tabular}{|c|c|c|c|c}
\hline Groups & $\begin{array}{c}\text { Mean R-R } \\
\text { interval(Sec) }\end{array}$ & $\begin{array}{c}\text { Mean HR } \\
(\mathbf{b p m})\end{array}$ & $\begin{array}{c}\text { SDNN } \\
(\mathbf{m s})\end{array}$ & $\begin{array}{c}\text { RMSSD } \\
(\mathbf{m s})\end{array}$ \\
\hline $\mathrm{A}(\mathrm{n}=30)$ & $0.9 \pm 0.15$ & $71.13 \pm 7.37$ & $65.58 \pm 17.07$ & $32.33 \pm 4.9$ \\
\hline $\mathrm{B}_{1}(\mathrm{n}=30)$ & $0.79 \pm 0.2$ & $76.97 \pm 9.12$ & $49.82 \pm 6.38$ & $20.03 \pm 2.6$ \\
\hline $\mathrm{B}_{2}(\mathrm{n}=30)$ & $0.8 \pm 0.15$ & $72.70 \pm 8.75$ & $51.99 \pm 7.70$ & $23.37 \pm 3.1$ \\
\hline
\end{tabular}

Statistical analysis

\begin{tabular}{|c|c|c|c|c|}
\hline Groups & \multicolumn{4}{|c|}{$p$ value } \\
\hline$A$ vs $B_{1}$ vs $B_{2 a}$ & $0.007^{* *}$ & $0.025^{*}$ & $0.000^{\star * *}$ & $0.000^{* *}$ \\
\hline$A$ vs $B_{1 b}$ & $0.003^{* *}$ & $0.008^{* *}$ & $0.000^{\star * *}$ & $0.000^{* *}$ \\
\hline$A$ vs $B_{2 b}$ & 0.019 * & $0.456 \mathrm{~ns}$ & $0.000^{* * *}$ & $0.000^{* *}$ \\
\hline$B_{1}$ vs $B_{2 b}$ & $0.648 \mathrm{~ns}$ & $0.070 \mathrm{~ns}$ & $0.239 \mathrm{~ns}$ & $0.000^{* *}$ \\
\hline
\end{tabular}

Data were expressed as mean $\pm S D$. Figures in parentheses indicate ranges. Statistical analyses were done by One-way ANOVA ${ }^{a}$ and Independent sample t-test ${ }^{\mathrm{b}}$. 
$\mathrm{R}-\mathrm{R}=$ Interval between successive QRS complex (sec), $\mathrm{HR}=$ Heart rate,

SDNN=Standard deviation of NN interval,

$\mathrm{NN}=$ Intervals between adjacent QRS complexes from SA node depolarization.

RMSSD=Square root of mean squared differences between adjacent $\mathrm{NN}$ intervals,

$\mathrm{ms}_{1}=$ millisecond, $\mathrm{ms}_{2}=$ squared millisecond .

$* * *=p<0.001,{ }^{*}=<0.05, n s=p>0.05$,

$\mathrm{n}=$ number of subjects

The mean total power $(p<0.001)$, LF power $(p<0.01)$ and HF power $(p<0.001)$ were significantly lower in both groups $B_{1}$ and $B_{2}$ than that of group $A$. However, no significant difference was observed between group $B_{1}$ vs group $B_{2}$ (Table-IV). Again LF norm and LF/HF ratio were significantly $(p<0.001)$ higher and HF norm were significantly $(p<0.001)$ lower in both groups $B_{1}$ and $B_{2}$ when compared to those of group $A$ and also in $B_{1}$ compared to that of $\mathrm{B}_{2}$ (Table-IV).

Table-IV: Frequency domain measures of HRV in different groups $(n=90)$

\begin{tabular}{|c|c|c|c|}
\hline Groups & Total power(ms $)$ & LF power(ms $\left.{ }^{2}\right)$ & HF power(ms $\left.{ }^{2}\right)$ \\
\hline$A(n=30)$ & $2815.4 \pm 813.8$ & $435 \pm 347.6$ & $621 \pm 606.5$ \\
\hline $\mathrm{B}_{1}(\mathrm{n}=30)$ & $1487.4 \pm 291.2$ & $196.1 \pm 179.7$ & $49.7 \pm 44.4$ \\
\hline $\mathrm{B}_{2}(\mathrm{n}=30)$ & $1674.8 \pm 437.3$ & $185 \pm 171.6$ & $77.2 \pm 74.1$ \\
\hline
\end{tabular}

\begin{tabular}{|c|c|c|c|}
\hline \multicolumn{4}{|c|}{ Statistical analysis } \\
\hline Groups & & P value & \\
\hline$A$ vs $B_{1}$ vs $B_{2 a}$ & $0.000^{* * *}$ & $0.000^{* \star *}$ & $0.000^{* * *}$ \\
\hline$A$ vs $B_{1 b}$ & $0.000^{* * *}$ & $0.001^{\star *}$ & $0.000^{* * *}$ \\
\hline$A$ vs $B_{2 b}$ & $0.000^{* * *}$ & $0.001^{* *}$ & $0.000^{* * *}$ \\
\hline$B_{1}$ vs $B_{2 b}$ & $0.056 n s$ & $0.808 \mathrm{~ns}$ & $0.087 \mathrm{~ns}$ \\
\hline
\end{tabular}

Data were expressed as mean $\pm S D$. Figures in parentheses indicate ranges. Statistical analysis were done by One-way ANOVA ${ }^{a}$ and Independent sample t-test ${ }^{b}$, LF=Low Frequency, HF=High Frequency.

Table-V: Frequency domain measures of HRV in different groups $(n=90)$

\begin{tabular}{|c|c|c|c|}
\hline Groups & LF norm(nu) & HF norm(nu) & LF/HF \\
\hline$A(n=30)$ & $59.70 \pm 3.65$ & $38.91 \pm 4.70$ & $1.5 \pm 0.23$ \\
\hline$B 1(n=30)$ & $80.56 \pm 3.29$ & $19.44 \pm 3.29$ & $4.32 \pm 1.06$ \\
\hline$B 2(n=30)$ & $71.13 \pm 4.75$ & $28.87 \pm 4.75$ & $2.55 \pm 0.57$ \\
\hline
\end{tabular}

Statistical analysis

\begin{tabular}{|c|l|l|l|}
\hline Groups & \multicolumn{3}{|c|}{ p value } \\
\hline $\mathrm{A}_{\text {vs }} \mathrm{B}_{1}$ vs $\mathrm{B}_{2 \mathrm{a}}$ & $0.000^{* * *}$ & $0.000^{* * *}$ & $0.000^{* * *}$ \\
\hline $\mathrm{A}$ vs $\mathrm{B}_{1 \mathrm{~b}}$ & $0.000^{* * *}$ & $0.000^{* * *}$ & $0.000^{* * *}$ \\
\hline $\mathrm{A}$ vs $\mathrm{B}_{2 \mathrm{~b}}$ & $0.000^{* * *}$ & $0.000^{* * *}$ & $0.000^{* * *}$ \\
\hline $\mathrm{B}_{1}$ vs $\mathrm{B}_{2 \mathrm{~b}}$ & $0.000^{* * *}$ & $0.000^{* * *}$ & $0.000^{* * *}$ \\
\hline
\end{tabular}

Data were expressed as mean $\pm S D$. Figures in parentheses indicate ranges, $L F$ norm $=L F$ power in normalized unit [(LF/Total power-VLF) x100],

HF norm = High frequency in normalized unit [(HF/Total power-VLF) x100],

$\mathrm{LF} / \mathrm{HF}=$ ratio of low and high frequency power.

${ }^{* * *}=p<0.001, \quad n=$ number of subjects.

\section{Discussion}

The present study was carried out to observe the heart rate variability (HRV) in male patients with hypertension in order to assess their cardiac autonomic nervous activity. HRV parameters were measured like LF power and LF norm for sympathetic and mean $R-R$ interval, mean heart rate, SDNN, RMSSD, total power, HF power and HF norm for parasympathetic and LF/HF ratio for sympathovagal balance. Findings of HRV parameters in healthy control group were almost similar to those reported by the various investigators from different countries ${ }^{9,10,12,13}$ and also from our country ${ }^{18,19,20}$.

In this study, the mean resting pulse rate and blood pressure were significantly higher in untreated hypertensive patients in comparison to those of healthy normotensive and treated hypertensive groups. But these values showed no statistical significant differences between healthy normotensive and treated hypertensive groups. Similar findings were also observed by various reporters of different countries $^{3,9,12,14,18}$. The mean R-R interval and the mean values of SDNN and RMSSD were observed in untreated hypertensive patients than those of healthy control. Similar significant changes in these parameters in hypertensive patients were also reported by by investigators of many countries ${ }^{8-16}$.

The LF norm and LF/HF ratio were significantly higher and HF norm power was significantly lower in both groups of hypertensive patients than those of healthy controls. Again, untreateded hypertensive patients had significantly higher values of LF norm and LF/HF ratio and significantly lower HF norm than those of treated groups. Almost similar type of findings were also reported by investigators of different countries ${ }^{3,7,11,12,14,21,22}$.

Researchers of different countries have suggested that essential hypertension is commonly neurogenic and attributed to sympathetic overdrive. They also reported that several factors are responsible for the changes of autonomic nerve function activities associated with essential hypertension. Presence of autonomic nerve dysfunction may lead to arterial hypertension ${ }^{8,10,13,22}$. In these cases there is increased rate of sympathetic nerve firing and also increased density of sympathetic innervations. Moreover co-transmission of adrenaline in cardiac 
sympathetic nerves along with impaired removal of noradrenaline from the synaptic cleft may also be the contributory factors for sympathetic over activity in essential hypertension ${ }^{4}$. Again some investigators recommended that $\beta$ adrenergic mediated vasodilation may have some contribution in the sympathetic overdrive in essential hypertension ${ }^{23-25}$.

In the present study, sympathetic overactivity may be the cause of hypertension as evidenced by the higher values of LF norm and LF/HF. However, decreased values of SDNN, RMSSD, total power, HF power and HF norm in hypertensive patients are suggestive of reduced vagal tone. All these changes indicate shifting of sympathovagal balance towards sympathetic predominance which is also in favour of sympathetic overactivity in essential hypertension.

\section{Conclusion}

Sympathovagal imbalance may occur in hypertensive patients and is usually characterized by both higher sympathetic and lower vagal modulation of the heart rate and effective treatment of the patients may return the balance towards normal.

\section{References}

1. Shahriar I, Haque Z, Momen A. Patterns of Antihypertensive Drugs Used in Two Postgraduate Institutions. Journal of Dhaka National Medical college and Hospital 2008; 13(1):41-4.

2. Wu J, Lu F, Yang Y et al. Epidemiological Study on the Effect of Pre hypertension and Family History of Hypertension on Cardiac Autonomic Function. J Am Coll Cardiol 2008; 51:1896-1901.

3. Julius S. Autonomic nervous system dysregulation in human hypertension. Am J Cardiol 1991; 67:3B-7B.

4. Esler M, Rumantir M, Kaye D et al. Sympathetic nerve biology in essential hypertension. ClinExp Pharmacol Physiol 2001; 28(12):986-9.

5. Pagani M, Lucini D. Autonomic dysregulation in essential hypertension: Insight from heart rate and arterial pressure variability. AutonNeurosci 2001; 90:76-82.

6. Guzzetti S, Piccaluga E, Casati R et al. Sympathetic predominance in essential hypertension: A study employing spectral analysis of heart rate variability. J Hypertension 1988; 6:711-7.
7. Heart Rate Variability Testing. [Internet] 2008 Available from:http:///www.docgeorge.com.

8. Singh JP, Larson MG, Tsuji H et al. Reduced heart rate variability in new-onset hypertension. Insights into pathogenesis of hypertension. The Framingham Heart study. Hypertension 1998; 32:293-7.

9. Kaftan AH and Kaftan O. QT Intervals and Heart Rate Variability in Hypertensive Patients. Jpn Heart J 2000; 41(2):173-82.

10. Pavithra P, Madanmohan, Mithun R et al. Heart Rate Variability in Middle Aged Men with New-Onset Hypertension. Ann of Noninvasive Electrocardiol 2008; 13(3):242-8.

11. Sevre K, Johan D, Nordby G et al. Autonomic Function in Hypertensive and Normotensive Subjects: Importance of Gender. Hypertension 2001; 37:1351-6.

12. Mussalo $H$, Vanninen $E$, Ikäheimo $R$ et al. Heart rate variability and its determinants in patients with severe or mild essential hypertension. Clin Physiol 2008; 21(5):594-604.

13. Prakash ES, Madanmohan, Sethuraman KR et al. Cardiovascular autonomic regulation in subjects with normal blood pressure, high-normal blood pressure and recent-onset hypertension. ClinExp-Pharmacol Physiol 2005; 32:488-94.

14. Huikuri HV, Ylitalo A, Pikkujamasa SM et al. Heart rate variability in systemic hypertension. Am J Cardiol 1996; 77:1073-7.

15. Pikkujamsa SM, Huikuri HV, Airaksinen KE et al. Heart rate variability and baroreflex sensitivity in hypertensive subjects with and without metabolic features of insulin resistance syndrome. Am J Hypertens 1998;11:523-31.

16. Chakko S, Mulingtapang RF, Huikuri HV et al. Alterations in heart rate variability and its circadian rhythm in hypertensive patients with left ventricular hypertrophy free of coronary artery disease. Am Heart J 1993; 126:1364-72.

17. Dietrich FD, Schindler C, Schwartz J et al. Heart Rate Variability In Aging Population and Its Association With lifestyle and Cardiovascular Risk Factors: Results of the SAPALDIA study. Europace 2006; 8:521-9. 
18. Kabir MR, Begum N, Ferdousi S et al. Heart Rate Variability in Hyperthyroidism. J Bangladesh Soc Physiol 2009 Dec; 4(2):51-7. available at http:// www.banglajol.info/index.php/JBSP/article/view/4172.

19. Ahmed M, Begum N, Ferdousi S. Assessment of Autonomic Nerve Function In Hypothyroids By Time Domain Method of Heart Rate Variability. J Bangladesh Soc Physiol 2012 June; 7(1):48-52. available at http://www.banglajol.info/index.php/JBSP/article/view File/11162/8156.

20. Mithun S, Begum N, Ferdousi S. Time Domain Measures Of Heart Rate Variability In Heavy Workers. J Bangladesh Soc Physiol 2011 December; 6(2):77-83. available at http://www.banglajol.info/index.php/JBSP/ article/view/9755.

21. Nordby G, Os I, Kjeldsen SE et al. Mild essential hypertension in nonobese premenopausal women is characterized by low renin. Am J Hypertens 1992; 5:579-84.
22. Lucini D, Mela GS, Malliani A et al. Impairment in cardiac autonomic regulation preceding arterial hypertension in humans: insights from spectral analysis of beat-by-beat cardiovascular variability. PubMed 2002 Nov 19; 106(21):2673-9.

23. Feldman RD. Defective venous beta-adrenergic response in borderline hypertensive subjects is corrected by a low sodium diet. JClin Invest 1990; 85:647-52.

24. Naslund T, Silberstein DJ, Merrell WJ et al. Low Sodium Intake Corrects Abnormality In-ReceptorMediated Arterial Vasodilation In Patients With Hypertension: Correlation With-Receptor Function In Vitro. Clin Pharmacol Ther 1990; 48:87-95.

25. Stein CM, Nelson R, Deegan $\mathrm{R}$ et al. Fore arm Beta Adrenergic Receptor- Mediated Vasodilation Is Impaired Without Alteration Of Forearm Norepinephrine Spillover In Borderline Hypertension. J Clin Invest 1995; 96:579-85. 